\title{
ALGEBRAIC HULLS OF SOLVABLE GROUPS AND EXPONENTIAL ITERATED INTEGRALS ON SOLVMANIFOLDS
}

\author{
HISASHI KASUYA
}

\begin{abstract}
We represent the coordinate ring of algebraic hulls (which are generalizations of the Malcev completions of nilpotent groups for solvable groups) of solvmanifolds $G / \Gamma$ by using Miller's exponential iterated integrals (which are extensions of Chen's iterated integrals) of invariant differential forms.
\end{abstract}

\section{Introduction}

Let $G$ be a simply connected Lie group and $\mathfrak{g}$ the Lie algebra of $G$. We consider the space $\wedge \mathfrak{g}_{\mathbb{C}}^{*}$ of $\mathbb{C}$-valued left $G$-invariant differential forms on $G$. We assume that $G$ has a lattice (i. e. cocompact discrete subgroup) $\Gamma$. We consider the compact homogeneous space $G / \Gamma$ and $\bigwedge \mathfrak{g}_{\mathbb{C}}^{*}$ as a subcomplex of the de Rham complex $A_{\mathbb{C}}^{*}(G / \Gamma)$ of $G / \Gamma$. Suppose $G$ is nilpotent. Then we have the unique unipotent algebraic group $\mathbf{U}_{\Gamma}$ called the Malcev completion of $\Gamma$ such that there is a injection $\Gamma \rightarrow \mathbf{U}_{\Gamma}$ with the Zariski-dense image. We can represent the coordinate ring of $\mathbf{U}_{\Gamma}$ by using Chen's iterated integrals on $G / \Gamma$ (see [2]). Since the inclusion $\wedge \mathfrak{g}_{\mathbb{C}}^{*} \subset A_{\mathbb{C}}^{*}(G / \Gamma)$ induces a cohomology isomorphism by Nomizu's theorem [11], $\bigwedge \mathfrak{g}_{\mathbb{C}}^{*}$ is the Sullivan minimal model of $A_{\mathbb{C}}^{*}(G / \Gamma)$ (see [4). This implies $H^{0}\left(\bar{B}\left(\bigwedge \mathfrak{g}_{\mathbb{C}}^{*}\right)\right) \cong H^{0}\left(\bar{B}\left(A_{\mathbb{C}}^{*}(G / \Gamma)\right)\right)$ where $\bar{B}\left(\bigwedge \mathfrak{g}_{\mathbb{C}}^{*}\right)$ and $\bar{B}\left(A_{\mathbb{C}}^{*}(G / \Gamma)\right)$ are the reduced bar constructions of $\bigwedge \mathfrak{g}_{\mathbb{C}}^{*}$ and $A_{\mathbb{C}}^{*}(G / \Gamma)$ respectively (see [3]). Hence we can represent the coordinate ring of $\mathbf{U}_{\Gamma}$ by using Chen's iterated integrals of left-invariant forms.

Suppose $G$ is solvable. Then Chen's iterated integrals on $G / \Gamma$ does not give sufficient information on the fundamental group of $G / \Gamma$. For example, let $G=$ $\mathbb{R} \ltimes_{\phi} \mathbb{R}^{2}$ such that $\phi(t)=\left(\begin{array}{cc}e^{t} & 0 \\ 0 & e^{-t}\end{array}\right)$. Then $G$ has a lattice $\Gamma$ and the inclusion $\bigwedge \mathfrak{g}_{\mathbb{C}}^{*} \subset A_{\mathbb{C}}^{*}(G / \Gamma)$ induces a cohomology isomorphism (see [5]). Since we have $H^{1}(G / \Gamma, \mathbb{C})=H^{1}\left(\bigwedge \mathfrak{g}_{\mathbb{C}}^{*}\right)=\mathbb{C}$, by Chen's results, iterated integrals represent the coordinate ring of a additive algebraic group $\mathbb{G}_{a d}=\mathbb{C}$ (see [7). But since $\Gamma$ is solvable and not abelian, we can't embed $\Gamma$ in $\mathbb{G}_{\text {ad }}$.

In 10, as an extension of the Malcev completion, Mostow constructed a Zariskidense embedding of $\Gamma$ in an algebraic group $\mathbf{H}_{\Gamma}$ called algebraic hull of $\Gamma$. In [7, Miller gave extensions of Chen's iterated integrals called exponential iterated integrals. In this paper we represent the coordinate ring of $\mathbf{H}_{\Gamma}$ by using Miller's exponential iterated integrals of left-invariant differential forms on $G / \Gamma$.

\section{Relative completions and algebraic hulls}

Let $G$ be a discrete group (resp. a Lie group). We call a map $\rho: G \rightarrow G L_{n}(\mathbb{C})$ a representation, if $\rho$ is a homomorphism of groups (resp. Lie groups). In this paper

Key words and phrases. exponential iterated integral, algebraic hull, solvmanifold. 
we denote by $T_{n}(\mathbb{C})$ the group of $n \times n$ upper triangular matrix and denote by $U_{n}(\mathbb{C})$ the group of $n \times n$ upper triangular unipotent matrix.

2.1. algebraic groups and pro-algebraic groups. In this paper an algebraic group means an affine algebraic variety $\mathbf{G}$ over $\mathbb{C}$ with a group structure such that the multiplication and inverse are morphisms of varieties. All algebraic groups arise as Zariski-closed subgroups of $G L_{n}(\mathbb{C})$. A pro-algebraic group is an inverse limit of algebraic groups. If a pro-algebraic group is an inverse limit of unipotent algebraic groups, it is called pro-unipotent. Let $\mathbf{G}$ be a pro-algebraic group. We denote by $\mathbf{U}(\mathbf{G})$ the maximal pro-unipotent normal subgroup called the pro-unipotent radical. If $\mathbf{U}(\mathbf{G})=e, \mathbf{G}$ is called reductive. We denote by $\mathbb{C}[\mathbf{G}]$ the coordinate ring of $\mathbf{G}$. The group structure on $\mathbf{G}$ induces a Hopf algebra structure on $\mathbb{C}[\mathbf{G}]$. It is known that we have the anti-equivalence between algebras and affine varieties induces an anti-equivalence between pro-algebraic groups and reduced Hopf algebras.

Theorem 2.1. ([9, [6]) Let $\mathbf{G}$ be a pro-algebraic group. Then the exact sequence

$$
1 \longrightarrow \mathbf{U}(\mathbf{G}) \longrightarrow \mathbf{G} \longrightarrow \mathbf{G} / \mathbf{U}(\mathbf{G}) \longrightarrow 1
$$

splits.

Let $G$ be a discrete group or Lie group. We denote by $A(G)$ the inverse limit of all representations $\phi: G \rightarrow \mathbf{G}$ with Zariski-dense images. We call the pro-unipotent radical $\mathbf{U}(A(G))$ of $A(G)$ the unipotent hull of $G$ and denote it $\mathbf{U}_{G}$.

2.2. Relative completion. Let $\rho: G \rightarrow \mathbf{S}$ be a representation of $G$ to a diagonal algebraic group $\mathbf{S}$ with the Zariski-dense image. Let $\phi: G \rightarrow \mathbf{G}$ be a representation of $G$ to an algebraic group $\mathbf{G}$ with the Zariski-dense image. We call $\phi$ a $\rho$-relative representation if we have the commutative diagram

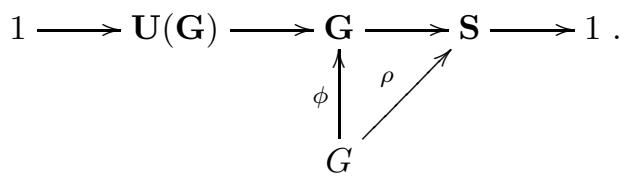

If $\mathbf{S}$ is contained in an algebraic torus, for any $\rho$-relative representation $\phi$ : $G \rightarrow \mathbf{G}$ there exists a faithful representation $\mathbf{G} \hookrightarrow T_{n}(\mathbb{C})$ such that $\mathbf{G} \cap U_{n}(\mathbb{C})=$ $\mathbf{U}(\mathbf{G})$ (see [7]).

We denote by $\mathcal{G}_{\rho}(G)$ the inverse limit of $\rho$-relative representations $\phi_{i}: G \rightarrow \mathbf{G}_{i}$. We call $\mathcal{G}_{\rho}(G)$ the $\rho$-relative completion of $G$. If $\mathbf{S}$ is trivial, $\mathcal{G}_{\rho}(G)$ is the classical Malcev (or unipotent) completion.

2.3. Algebraic hulls. We define the algebraic hulls of polycyclic groups (resp. Lie groups) which constructed in [10].

A group $\Gamma$ is polycyclic if it admits a sequence

$$
\Gamma=\Gamma_{0} \supset \Gamma_{1} \supset \cdots \supset \Gamma_{k}=\{e\}
$$

of subgroups such that each $\Gamma_{i}$ is normal in $\Gamma_{i-1}$ and $\Gamma_{i-1} / \Gamma_{i}$ is cyclic. For a polycyclic group $\Gamma$, we denote $\operatorname{rank} \Gamma=\sum_{i=1}^{i=k} \operatorname{rank} \Gamma_{i-1} / \Gamma_{i}$. Let $G$ be a simply connected solvable Lie group and $\Gamma$ be a lattice of $G$. Then $\Gamma$ is torsion-free polycyclic and $\operatorname{dim} G=\operatorname{rank} \Gamma$.

Let $G$ be a simply connected solvable Lie group or torsion-free polycyclic group. Consider the algebraic completion $A(G)$. Then it is known that the unipotent hull 
$\mathbf{U}_{G}=\mathbf{U}(A(G))$ is finite dimensional (see [10]). By Theorem 2.1] we have a splitting $A(G)=\left(A(G) / \mathbf{U}_{G}\right) \ltimes_{\phi} \mathbf{U}_{G}$. Let $K$ be the kernel of the action $\phi:\left(A(G) / \mathbf{U}_{G}\right) \rightarrow$ $\operatorname{Aut}\left(\mathbf{U}_{G}\right)$. Then $K$ is the maximal reductive normal subgroup of $A(G)$ (see [10]). Denote $\mathbf{H}_{G}=A(G) / K$ and call it the algebraic hull of $G$.

Theorem 2.2. ([10, [13]) Let $G$ be a simply connected solvable Lie group (resp. torsion-free polycyclic group). Then $G \rightarrow \mathbf{H}_{G}$ is injective and $\mathbf{H}_{G}$ is a finite dimensional algebraic group such that:

(1) $\operatorname{dim} \mathbf{U}\left(\mathbf{H}_{G}\right)=\operatorname{dim} G($ resp. $=\operatorname{rank} G)$.

(2) The centralizer of $\mathbf{U}\left(\mathbf{H}_{G}\right)$ in $\mathbf{H}_{G}$ is contained in $\mathbf{U}\left(\mathbf{H}_{G}\right)$.

Conversely if an algebraic group $\mathbf{H}$ with an injective homomorphism $\psi: G \rightarrow \mathbf{H}$ with the Zariski-dense image satisfies the properties (1) and (2), then $\mathbf{H}$ is isomorphic to $\mathbf{H}_{G}$.

2.4. Direct constructions of algebraic hulls. The idea of this subsection is based on classical works of semi-simple splitting (see [14, 12] and the references given there). Let $\mathfrak{g}$ be a solvable Lie algebra, and $\mathfrak{n}=\left\{X \in \mathfrak{g} \mid \operatorname{ad}_{X}\right.$ is nilpotent $\}$. $\mathfrak{n}$ is the maximal nilpotent ideal of $\mathfrak{g}$ and called the nilradical of $\mathfrak{g}$. Then we have $[\mathfrak{g}, \mathfrak{g}] \subset \mathfrak{n}$. Let $D(\mathfrak{g})$ be the Lie algebra of derivations of $\mathfrak{g}$. By the Jordan decomposition, we have $\operatorname{ad}_{X}=\operatorname{ad}_{s X}+\operatorname{ad}_{n X}$ such that $\operatorname{ad}_{s X}$ is a semi-simple operator and $\operatorname{ad}_{n X}$ is a nilpotent operator. Since we have $d_{X}, n_{X} \in D(\mathfrak{g})$, we have the $\operatorname{map~ad}_{s}: \mathfrak{g} \rightarrow D(\mathfrak{g})$. Since ad is trigonalizable (Lie's theorem), this map is homomorphism with the kernel $\mathfrak{n}$. Let $\overline{\mathfrak{g}}=\operatorname{Im}_{\operatorname{ad}_{s}} \ltimes \mathfrak{g}$. and $\overline{\mathfrak{n}}=\left\{X-\operatorname{ad}_{s X} \in \overline{\mathfrak{g}} \mid X \in\right.$ $\mathfrak{g}\}$.

Proposition 2.3. $\overline{\mathfrak{n}}$ is a nilpotent ideal of $\overline{\mathfrak{g}}$ and we have a decomposition $\overline{\mathfrak{g}}=$ $\operatorname{Im}_{\operatorname{ad}} \ltimes \overline{\mathfrak{n}}$.

Proof. By $\operatorname{ad}_{X-\operatorname{ad}_{s X}}=\operatorname{ad}_{X}-\operatorname{ad}_{s X}$ on $\mathfrak{g}, \operatorname{ad}_{X-\operatorname{ad}_{s X}}$ is a nilpotent operator and hence $\overline{\mathfrak{n}}$ consists of nilpotent elements. By Lie's theorem, we have a basis

$$
X_{1}, \ldots, X_{l}, X_{l+1} \ldots, X_{n}
$$

of $\mathfrak{g} \otimes \mathbb{C}$ such that ad is represented by upper triangular matrices. Since the nilradical $\mathfrak{n}$ is an ideal, $\mathfrak{n} \otimes \mathbb{C}$ is ad-invariant subspace of $\mathfrak{g} \otimes \mathbb{C}$. We choose $X_{1}, \ldots, X_{l}$ a basis of $\mathfrak{n} \otimes \mathbb{C}$. By $[\mathfrak{g}, \mathfrak{g}] \subset \mathfrak{n}$, we have $\operatorname{ad}_{X}(\mathfrak{g} \otimes \mathbb{C}) \subset \mathfrak{n} \otimes \mathbb{C}=\left\langle X_{1}, \ldots, X_{l}\right\rangle$, and hence ad represented as

$$
\operatorname{ad}_{X}=\left(\begin{array}{cccccc}
a_{11}(X) & \ldots & & & \ldots & a_{1 l}(X) \\
& \ddots & & & & \vdots \\
& & a_{l l}(X) & \ldots & & a_{l m}(X) \\
& & & 0 & \ldots & 0 \\
& & & & \ddots & \vdots \\
& & & & & 0
\end{array}\right) .
$$

Thus we have $\operatorname{ad}_{s X}\left(X_{i}\right)=a_{11}(X) X_{i}$ for $1 \leq i \leq l$ and $\operatorname{ad}_{s X}\left(X_{i}\right)=0$ for $l+1 \leq$ $i \leq n$. By this we have

$$
\left[X_{i}+\operatorname{ad}_{s Y}, X_{j}+\operatorname{ad}_{s Z}\right] \in\left\langle X_{1}, \ldots, X_{l}\right\rangle=\mathfrak{n} \otimes \mathbb{C}
$$

for any $1 \leq i, j \leq n, Y, Z \in \mathfrak{g}$. This implies $[\overline{\mathfrak{g}}, \overline{\mathfrak{g}}] \subset \mathfrak{n}$. By $\mathfrak{n} \subset \overline{\mathfrak{n}}, \overline{\mathfrak{n}}$ is an ideal of $\overline{\mathfrak{g}}$ and we have $\overline{\mathfrak{g}}=\left\{\operatorname{ad}_{s X}+Y-\operatorname{ad}_{s Y} \mid X, Y \in \mathfrak{g}\right\}=\operatorname{Imad}_{s} \ltimes \overline{\mathfrak{n}}$. 
By this proposition, we have the inclusion $i: \mathfrak{g} \rightarrow D(\overline{\mathfrak{n}}) \ltimes \overline{\mathfrak{n}}$ given by $i(X)=$ $\operatorname{ad}_{s X}+X-\operatorname{ad}_{s X}$ for $X \in \mathfrak{g}$.

Let $G$ be a simply connected solvable Lie group and $\mathfrak{g}$ be the Lie algebra of $G$. For the adjoint representation Ad $: G \rightarrow \operatorname{Aut}(\mathfrak{g})$, we consider the semi-simple part $\operatorname{Ad}_{s}: G \rightarrow \operatorname{Aut}(\mathfrak{g})$ as similar to the Lie algebra case. Denote by $T$ the universal covering of $\operatorname{Ad}_{s}(G)$. Let $\bar{N}$ be the simply connected Lie group which corresponds to $\overline{\mathfrak{n}}$. Then by Proposition 2.3, we have $T \ltimes G=T \ltimes \bar{N}$. By the proof of this proposition, the action $T \rightarrow \operatorname{Aut}(\overline{\mathfrak{n}})$ is the extension of the action of $\operatorname{Im}_{\operatorname{ad}}$. Hence we have $\operatorname{Ad}_{s}(G) \ltimes G=\operatorname{Ad}_{s}(G) \ltimes \bar{N}$.

A simply connected nilpotent Lie group is considered as the real points of a unipotent $\mathbb{R}$-algebraic group (see [12, p. 43]) by the exponential map. We have the unipotent $\mathbb{R}$-algebraic group $\overline{\mathbf{N}}$ with $\overline{\mathbf{N}}(\mathbb{R})=\bar{N}$. We identify $\operatorname{Aut}_{\mathrm{a}}(\overline{\mathbf{N}})$ with $\operatorname{Aut}\left(\mathfrak{n}_{\mathbb{C}}\right)$ and $\operatorname{Aut}_{\mathrm{a}}(\overline{\mathbf{N}})$ has the $\mathbb{R}$-algebraic group structure with $\operatorname{Aut}_{a}(\overline{\mathbf{N}})(\mathbb{R})=\operatorname{Aut}(N)$. So we have the $\mathbb{R}$-algebraic group $\operatorname{Aut}_{\mathrm{a}}(\overline{\mathbf{N}}) \ltimes \overline{\mathbf{N}}$. Then by $\operatorname{Ad}_{s}(G) \ltimes G=\operatorname{Ad}_{s}(G) \ltimes \bar{N} \subset$ $\operatorname{Aut}_{\mathrm{a}}(\overline{\mathbf{N}}) \ltimes \overline{\mathbf{N}}$, we consider the Zariski-closure $\mathbf{G}$ of $G$ in $\operatorname{Aut}_{\mathrm{a}}(\overline{\mathbf{N}}) \ltimes \overline{\mathbf{N}}$. Since $\operatorname{Ad}_{s}(G)$ is a group of diagonal automorphisms, we have $\mathbf{U}(\mathbf{G})=\overline{\mathbf{N}} . \operatorname{By} \operatorname{dim} G=\operatorname{dim} \bar{N}$, we can easily check that $\mathbf{G}$ satisfies the properties (1), (2) in Theorem 2.2 and hence it is the algebraic hull $\mathbf{H}_{G}$ of $G$. Hence the inclusion $i: \mathfrak{g} \rightarrow D(\overline{\mathfrak{n}}) \ltimes \overline{\mathfrak{n}}$ induces the algebraic hull $I: G \rightarrow \mathbf{H}_{G}$ of $G$. Since $i: \mathfrak{g} \rightarrow D(\overline{\mathfrak{n}}) \ltimes \overline{\mathfrak{n}}$ is given by $i(X)=\operatorname{ad}_{s X}+X-\operatorname{ad}_{s X} \in D(\overline{\mathfrak{n}}) \ltimes \overline{\mathfrak{n}}$, the composition $G \rightarrow \mathbf{H}_{G} \rightarrow \mathbf{H}_{G} / \mathbf{U}\left(\mathbf{H}_{G}\right)$ is induced by the Lie algebra homomorphism $\operatorname{ad}_{s}: \mathfrak{g} \rightarrow D(\mathfrak{g})$ by $\mathbf{U}(\mathbf{G})=\overline{\mathbf{N}}$. Thus we have the following lemma.

Lemma 2.4. The algebraic hull $G \rightarrow \mathbf{H}_{G}$ is an $\mathrm{Ad}_{s}$-relative representation.

\subsection{Algebraic hulls and relative completions of solvable groups.}

Theorem 2.5. Let $G$ be a simply connected Lie group. Then the algebraic hull $\mathbf{H}_{G}$ is isomorphic to the $\operatorname{Ad}_{s}$-relative completion $\mathcal{G}_{\mathrm{Ad}_{s}}(G)$ of $G$.

Proof. Consider a commutative diagram

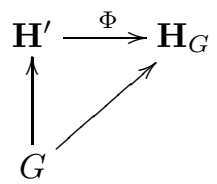

for some $\mathrm{Ad}_{s^{-}}$-relative representation $G \rightarrow \mathbf{H}^{\prime}$. Since $G \rightarrow \mathbf{H}^{\prime}$ and $G \rightarrow \mathbf{H}_{G}$ have Zariski-dense images, $\Phi: \mathbf{H}^{\prime} \rightarrow \mathbf{H}_{G}$ is surjective and the restriction $\Phi$ : $\mathbf{U}\left(\mathbf{H}^{\prime}\right) \rightarrow \mathbf{U}\left(\mathbf{H}_{G}\right)$ is also surjective. By $\mathbf{U}\left(\mathbf{H}_{G}\right)=\mathbf{U}_{G}, \Phi: \mathbf{U}\left(\mathbf{H}^{\prime}\right) \rightarrow \mathbf{U}\left(\mathbf{H}_{G}\right)$ is an isomorphism. Since $G \rightarrow \mathbf{H}^{\prime}$ and $G \rightarrow \mathbf{H}_{G}$ are $\mathrm{Ad}_{s^{-}}$-relative representations, $\Phi$ induces the isomorphism $\mathbf{H}^{\prime} / \mathbf{U}\left(\mathbf{H}^{\prime}\right) \rightarrow \mathbf{H}_{G} / \mathbf{U}\left(\mathbf{H}_{G}\right)$. Hence $\Phi: \mathbf{H}^{\prime} \rightarrow \mathbf{H}_{G}$ is an isomorphism. By the definition of $\operatorname{Ad}_{s}$-relative completion of $G$, we have the theorem.

Theorem 2.6. Let $G$ be a simply connected solvable Lie group and $\Gamma$ a lattice of $G$. Then the algebraic hull $\mathbf{H}_{\Gamma}$ of $\Gamma$ is isomorphic to $\operatorname{Ad}_{\left.s\right|_{\Gamma}}$-relative completion $\mathcal{G}_{\operatorname{Ad}_{s \mid \Gamma}}(\Gamma)$ of $\Gamma$.

Proof. For the algebraic hull $\psi: G \rightarrow \mathbf{H}_{G}$ of $G$, we consider the Zariski-closure of $\psi(\Gamma)$ in $\mathbf{H}_{G}$. Then by $\operatorname{dim} G=\operatorname{rank} \Gamma$ we can easily check that this algebraic group satisfies (1) and (2) in Theorem 2.2 and hence it is the algebraic hull $\mathbf{H}_{\Gamma}$ of $\Gamma$. Вy 
the above theorem, $\Gamma \rightarrow \mathbf{H}_{\Gamma}$ is a $\operatorname{Ad}_{\left.s\right|_{\Gamma}}$-relative representation. As similar to the above proof, we have the theorem.

\section{EXPONENTIAL ITERATED INTEGRAL ON SOLVMANIFOLDS}

In this section we consider Miller's exponential iterated integrals. Let $M$ be a $C^{\infty}$-manifold and $\Omega_{x} M$ be a space of piecewise smooth loops $\lambda:[0,1] \rightarrow M$ with $\lambda(0)=x$. For 1 -forms $\omega_{1}, \ldots, \omega_{n} \in A_{\mathbb{C}}^{*}(M)$, the iterated integral $\int \omega_{1} \omega_{2} \cdots \omega_{n}$ : $\Omega_{x} M \rightarrow \mathbb{C}$ is defined by

$$
\int_{\lambda} \omega_{1} \omega_{2} \cdots \omega_{n}=\int_{0 \leq t_{1} \leq t_{2} \leq \cdot t_{n} \leq 1} F\left(t_{1}\right) F\left(t_{2}\right) \cdots F\left(t_{n}\right) d t_{1} d t_{2} \cdots d t_{n} \lambda \in \Omega_{x} M
$$

where $F_{i}(t) d t=\lambda^{*} \omega_{i} \in A^{1}([0,1])$. In [7], for $\delta_{1}, \delta_{2}, \cdots, \delta_{n}, \omega_{12}, \omega_{23}, \cdots, \omega_{n-1 n} \in$ $A_{\mathbb{C}}^{1}(M)$ Miller defined the exponential iterated integral $\int e^{\delta_{1}} \omega_{12} e^{\delta_{2}} \omega_{23} \cdots e^{\delta_{n-1}} \omega_{n-1 n} e^{\delta_{n}}:$ $\Omega_{x} M \rightarrow \mathbb{C}$ as

$$
\begin{gathered}
\int_{\lambda} e^{\delta_{1}} \omega_{12} e^{\delta_{2}} \omega_{23} \cdots e^{\delta_{n-1}} \omega_{n-1 n} e^{\delta_{n}} \\
=\sum_{m_{1}, m_{2}, \cdots m_{n} \geq 0} \int_{\lambda} \underbrace{\delta_{1} \ldots \delta_{1}}_{m_{1} \text { terms }} \omega_{12} \underbrace{\delta_{2} \ldots \delta_{2}}_{m_{2} \text { terms }} \cdots \omega_{n-1 n} \underbrace{\delta_{n} \ldots \delta_{n}}_{m_{n} \text { terms }} .
\end{gathered}
$$

Then this infinite sum converges (see [7]). Let $L \subset A_{\mathbb{C}}^{1}(M)$ be a finitely generated $\mathbb{Z}$-module of 1 -forms such that $d L=0$. We denote $E^{L}(M, x)$ the $\mathbb{C}$-vector space of functions on $\Omega_{x} M$ generated by

$$
\left\{\int e^{\delta_{1}} \omega_{12} \cdots \omega_{n-1 n} e^{\delta_{n}} \mid \delta_{1}, \cdots \delta_{n} \in L, \omega_{12}, \omega_{23}, \cdots, \omega_{n-1 n} \in A_{\mathbb{C}}^{*}(M)\right\} .
$$

If $I \in E^{L}(M, x)$ is constant on homotopy classes of loops $\lambda:[0,1] \rightarrow M$ relative to $\{0,1\}$, we call $I$ a closed exponential iterated integral. Let $H^{0}\left(E^{L}(M, x)\right)$ denote the subspace of closed exponential iterated integrals. Take a $\mathbb{Z}$-basis $\left\{\delta_{1}, \delta_{2}, \ldots, \delta_{n}\right\}$ of $L$. Then we have the diagonal representation $\rho: \pi_{1}(M, x) \rightarrow D_{n}(\mathbb{C})$ such that $\rho(\lambda)=\operatorname{diag}\left(\int_{\lambda} e^{\delta_{1}}, \ldots, \int_{\lambda} e^{\delta_{n}}\right)$ for $\lambda \in \pi_{1}(M, x)$. Consider the $\rho$-relative completion $\mathcal{G}_{\rho}\left(\pi_{1}(M, x)\right)$ of the fundamental group of $M$. Miller showed the following theorem.

Theorem 3.1. ([7, Theorem 6.1]) The space $H^{0}\left(E^{L}(M, x)\right)$ is a Hopf algebra and we have a Hopf algebra isomorphism

$$
H^{0}\left(E^{L}(M, x)\right) \cong \mathbb{C}\left[\mathcal{G}_{\rho}\left(\pi_{1}(M, x)\right)\right] .
$$

Remark 3.1. For any $\rho$-relative representation $\phi: \pi_{1}(M, x) \rightarrow \mathbf{G}$, Miller showed that $\phi$ is the monodromy of a flat connection $\omega$ on $M \times \mathbb{C}^{n}$ whose connection form is an upper triangular matrix. Then the monodromy of $\omega$ is given by $I+$ $\sum_{i=1}^{\infty} \int \underbrace{\omega \omega \cdots \omega}_{i \text { terms }}$ and its matrix entries are exponential iterated integrals. In the proof of Theorem 6.1 of [7, Miller showed that these matrix entries generate the coordinate ring $\mathbb{C}[\mathbf{G}]$.

Consider a simply connected solvable Lie group $G$ with a lattice $\Gamma$. Take a diagonalization of the semi-simple part $\operatorname{ad}_{s}$ of the adjoint representation ad on $\mathfrak{g}$. Write $\operatorname{ad}_{s}=\operatorname{diag}\left(\delta_{1}, \ldots, \delta_{n}\right)$ where $\delta_{1}, \ldots, \delta_{n}$ are characters of $\mathfrak{g}$. By $\delta_{1}, \ldots, \delta_{n} \in$ $\operatorname{Hom}(\mathfrak{g}, \mathbb{C})$, we regard $\delta_{1}, \ldots, \delta_{n}$ as left-invariant closed 1 -forms. Let $L$ be the $\mathbb{Z}$ module generated by $\delta_{1}, \ldots, \delta_{n}$. Consider the algebraic hull $\mathbf{H}_{\Gamma}$ of $\Gamma$. Since we have $\pi_{1}(G / \Gamma, x) \cong \Gamma$, by Theorem 2.6 we have: 
Corollary 3.2. We have a Hopf algebra isomorphism

$$
H^{0}\left(E^{L}(G / \Gamma, x)\right) \cong \mathbb{C}\left[\mathbf{H}_{\Gamma}\right] .
$$

Let $E^{L}\left(\mathfrak{g}_{\mathbb{C}}^{*}\right)$ denote the subvector space of $E^{L}(G / \Gamma, x)$ generated by

$$
\left\{\int e^{\delta_{1}} \omega_{12} \cdots \omega_{n-1 n} e^{\delta_{n}} \mid \delta_{1}, \cdots \delta_{n} \in L \quad \omega_{12}, \omega_{23}, \cdots, \omega_{n-1 n} \in \mathfrak{g}_{\mathbb{C}}^{*}\right\} .
$$

Studying the proof of [7, Lemma 5.1], we can see that $E^{L}\left(\mathfrak{g}_{\mathbb{C}}^{*}\right)$ is closed under the multiplication. We define the subring

$$
H^{0}\left(E^{L}\left(\mathfrak{g}_{\mathbb{C}}^{*}\right)\right)=E^{L}\left(\mathfrak{g}_{\mathbb{C}}^{*}\right) \cap H^{0}\left(E^{L}(G / \Gamma, x)\right)
$$

of $H^{0}\left(E^{L}(G / \Gamma, x)\right)$.

Theorem 3.3. We have $H^{0}\left(E^{L}\left(\mathfrak{g}_{\mathbb{C}}^{*}\right)\right)=H^{0}\left(E^{L}(G / \Gamma, x)\right)$.

Proof. Consider the algebraic hull $\psi: G \rightarrow \mathbf{H}_{G}$ of $G$. Since $\psi: G \rightarrow \mathbf{H}_{G}$ is $\operatorname{Ad}_{s^{-}}$ relative, we can assume $\mathbf{H}_{G} \subset T_{r}(\mathbb{C})$ and $U_{r}(\mathbb{C}) \cap \mathbf{H}_{G}=\mathbf{U}\left(\mathbf{H}_{G}\right)$ as in Section 2.2. Let $\psi_{*}: \mathfrak{g} \rightarrow \mathfrak{t}_{r}(\mathbb{C})$ be the derivative of $\psi$ where $\mathfrak{t}_{r}(\mathbb{C})$ is the Lie algebra of $T_{r}(\mathbb{C})$. We write

$$
\psi_{*}=\left(\begin{array}{ccccc}
\omega_{11} & \omega_{12} & \cdots & & \omega_{1 r} \\
& \ddots & \ddots & & \vdots \\
& & \ddots & & \\
& & & \ddots & \omega_{r-1 r} \\
& & & & \omega_{r r}
\end{array}\right)
$$

as we consider $\psi_{*} \in \operatorname{Hom}(\mathfrak{g}, \mathbb{C}) \otimes T_{r}(\mathbb{C})$. Then we have

$$
\left(d \psi_{*}-\psi_{*} \wedge \psi_{*}\right)(X, Y)=\psi_{*}([X, Y])-\left[\psi_{*}(X), \psi_{*}(Y)\right]=0
$$

for $X, Y \in \mathfrak{g}$. Hence we have the flat connection $d-\psi_{*}$ on the vector bundle $G \times \mathbb{C}^{r}$. Consider the parallel transport $T=I+\sum_{i=1}^{\infty} \int \psi_{*} \cdots \psi_{*}$ of this connection. Let $P_{e} G$ be the space of the paths $\gamma:[0,1] \rightarrow G$ with $\gamma(0)=e$ where $e$ is the identity element of $G$. We consider the spaces $P_{e} G / \sim$ of homotopy classes of $\gamma \in P_{e} G$ relative to $\{0,1\}$. Since $G$ is simply connected, we have $P_{e} G / \sim=G$. It is easily seen that the parallel transport $T$ on $P_{e} G / \sim=G$ is a homomorphism whose derivative is equal to $\psi_{*}$. Hence we can identify the parallel transport $T$ on $P_{e} G / \sim$ with the representation $\psi$. Since $\psi$ is $\operatorname{Ad}_{s}$-relative and the diagonal entries of $T$ are $\int e^{\omega_{11}}, \ldots, \int e^{\omega_{r r}}$, we have $\omega_{11}, \ldots, \omega_{r r} \in L$. By the proof of Theorem 2.6 the injection $\phi: \Gamma \rightarrow \mathbf{H}_{\Gamma}$ is the restriction of $\psi$ on $\Gamma$. Thus the representation $\phi$ is the monodromy $I+\sum_{i=1}^{\infty} \int \psi_{*} \cdots \psi_{*}$ of the left-invariant flat connection $d-\psi_{*}$ on the vector bundle $G / \Gamma \times \mathbb{C}^{r}$. By Remark 3.1, the ring $\mathbb{C}\left[\mathbf{H}_{\Gamma}\right]$ is generated by matrix entries of $I+\sum_{i=1}^{\infty} \int \psi_{*} \cdots \psi_{*}$. Hence the theorem follows from Corollary 3.2 .

\section{An Example and a remark}

Let $N$ be a simply connected nilpotent Lie group and $\mathfrak{n}$ the Lie algebra of $N$. We suppose that $G$ has a lattice $\Gamma$. Then we can represent the coordinate ring of the Malcev completion of $\Gamma$ by using Chen's iterated integral of left-invariant forms on $N$. In this paper we give another representation of the Malcev completion of the fundamental group of some nilmanifold. 
Consider the solvable Lie group $G=\mathbb{R} \ltimes_{\mu} \mathbb{C}^{2}$ such that $\mu(t)=\left(\begin{array}{cc}e^{i \pi t} & t e^{i \pi t} \\ 0 & e^{i \pi t}\end{array}\right)$. We have the lattice $\Gamma=2 \mathbb{Z} \ltimes(\mathbb{Z}+i \mathbb{Z})$. We consider the inclusion $\bigwedge \mathfrak{g}_{\mathbb{C}}^{*} \subset A^{*}(G / \Gamma)$. The map $H^{*}\left(\bigwedge \mathfrak{g}_{\mathbb{C}}^{*}\right) \rightarrow H^{*}(G / \Gamma, \mathbb{C})$ induced by this inclusion is injective (see [13]). By $\left(\bigwedge \mathfrak{g}_{\mathbb{C}}^{*}\right)^{0}=\mathbb{C}$ and $\left(\bigwedge \mathfrak{g}_{\mathbb{C}}^{*}\right)^{1} \cap d A^{0}(G / \Gamma)=0$, we have an isomorphism

$$
H^{0}\left(B\left(\bigwedge \mathfrak{g}_{\mathbb{C}}^{*}, x\right)\right) \cong H^{0}\left(\bar{B}\left(\bigwedge \mathfrak{g}_{\mathbb{C}}^{*}\right)\right)
$$

where $H^{0}\left(B\left(\wedge \mathfrak{g}_{\mathbb{C}}^{*}, x\right)\right)$ is the space of closed Chen's iterated integrals of the leftinvariant forms on the based loop space $\Omega_{x} G / \Gamma$ and $H^{0}\left(\bar{B}\left(\bigwedge \mathfrak{g}_{\mathbb{C}}^{*}\right)\right)$ is the reduced bar construction (see [2]). Since we have $H^{1}\left(\bigwedge \mathfrak{g}_{\mathbb{C}}^{*}\right) \cong \mathbb{C}$, we have an isomorphism $H^{0}\left(B\left(\bigwedge \mathfrak{g}_{\mathbb{C}}^{*}, x\right)\right) \cong \mathbb{C}\left[\mathbb{G}_{\mathrm{ad}}\right]$

On the other hand, let $L$ be the sub $\mathbb{Z}$-module of $\mathfrak{g}_{\mathbb{C}}^{*}$ generated by $\{i \pi d t\}$. Then by Corollary 3.2 and Theorem 3.3 , we have an isomorphism

$$
H^{0}\left(E^{L}\left(\mathfrak{g}_{\mathbb{C}}^{*}\right)\right) \cong \mathbb{C}\left[\mathbf{H}_{\Gamma}\right] .
$$

Since we have $\mu(2 t)=\left(\begin{array}{cc}1 & 2 t \\ 0 & 1\end{array}\right)$ for $t \in \mathbb{Z}, \Gamma$ is nilpotent. Hence $\mathbf{H}_{\Gamma}$ is the Malcev completion of $\Gamma$. Since two compact solvmanifolds having the same fundamental group are diffeomorphic (see [8] or [13]), $G / \Gamma$ is diffeomorphic to a nilmanifold. By these arguments we notice:

Remark 4.1. By closed Chen's iterated integrals of the 1 -forms $\mathfrak{g}_{\mathbb{C}}^{*}$ on $G / \Gamma$, we can not represent the coordinate ring of Malcev completion of the fundamental group of the nilmanifold $G / \Gamma$. But the closed L-exponential iterated integrals of $\mathfrak{g}_{\mathbb{C}}^{*}$ enable us to represent it.

\section{Acknowledgements.}

The author would like to express his gratitude to Toshitake Kohno for helpful suggestions and stimulating discussions. This research is supported by JSPS Research Fellowships for Young Scientists.

\section{REFERENCES}

[1] A. Borel, Linear algebraic groups 2nd enl. ed Springer-verlag (1991).

[2] K. T. Chen, Iterated path integrals. Bull. Amer. Math. Soc. 83 (1977), no. 5, 831-879.

[3] K. T. Chen, Reduced Bar constructions on de Rham complexes, in: A. Heller, A. Tierney (eds), Algebra, Topology, and Category Theory, Academic Press, 1977, 19-32.

[4] K. Hasegawa, Minimal models of nilmanifolds. Proc. Amer. Math. Soc. 106 (1989), no. 1, $65-71$.

[5] A. Hattori, Spectral sequence in the de Rham cohomology of fibre bundles. J. Fac. Sci. Univ. Tokyo Sect. I 81960 289-331 (1960).

[6] G. Hochschild, G. D. Mostow, Pro-affine algebraic groups. Amer. J. Math. 911969 1127-1140.

[7] C. Miller, Exponential iterated integrals and the relative solvable completion of the fundamental group of a manifold, Topology 44(2005)351-373.

[8] G. D. Mostow, Factor spaces of solvable groups. Ann. of Math. (2) 60, (1954). 1-27.

[9] G. D. Mostow, Fully reducible subgroups of algebraic groups. Amer. J. Math. 78 (1956), 200-221.

[10] G. D. Mostow, Representative functions on discrete groups and solvable arithmetic subgroups. Amer. J. Math. 921970 1-32.

[11] K. Nomizu, On the cohomology of compact homogeneous spaces of nilpotent Lie groups. Ann. of Math. (2) 59, (1954). 531-538.

[12] A. L. Onishchik, and E. B. Vinberg, Discrete subgroups of Lie groups and cohomologies of Lie groups and Lie algebras, Springer (2000).

[13] M.S. Raghunathan, Discrete subgroups of Lie Groups, Springer-verlag, New York, 1972. 
[14] B. E. Reed, Representations of solvable Lie algebras, Michigan Math. J. 161969 227-233.

(H.kasuya) Graduate school of Mathematical SCIENCE UNIVERSity OF TOKYo JAPAN

E-mail address: khsc@ms.u-tokyo.ac.jp 